\title{
ASYMPTOTIC BEHAVIOR OF SOLUTIONS OF SECOND ORDER DIFFERENCE EQUATIONS WITH DEVIATING ARGUMENT
}

\author{
JANUSZ MIGDA AND MAGDALENA NOCKOWSKA-ROSIAK
}

Received 04 November, 2018

\begin{abstract}
We consider nonlinear second order difference equations with deviating argument of the form

$$
\Delta\left(r_{n} \Delta x_{n}\right)=a_{n} f\left(x_{n+\sigma-1}, x_{n+\sigma-2}, \ldots, x_{n+\sigma-m}\right)+b_{n} .
$$

We present sufficient conditions for the existence of solutions with prescribed asymptotic behavior. Moreover, we study the asymptotic behavior of solutions. We use $\mathrm{o}\left(n^{s}\right)$, for a given nonpositive real $s$, as a measure of approximation.
\end{abstract}

2010 Mathematics Subject Classification: 39A10; 39A22

Keywords: difference equation, prescribed asymptotic behavior, bounded solution, convergent solution, quasidifference, deviating argument

\section{INTRODUCTION}

In this paper we consider the nonlinear second order difference equation with deviating argument of the form

$$
\begin{gathered}
\Delta\left(r_{n} \Delta x_{n}\right)=a_{n} f\left(x_{n+\sigma-1}, x_{n+\sigma-2}, \ldots, x_{n+\sigma-m}\right)+b_{n} \\
r_{n}, a_{n}, b_{n} \in \mathbb{R}, \quad r_{n}>0, \quad \sigma \in \mathbb{Z}, \quad m \in \mathbb{N}, \quad f: \mathbb{R}^{m} \rightarrow \mathbb{R} .
\end{gathered}
$$

Here $\mathbb{N}, \mathbb{Z}, \mathbb{R}$ denote the set of positive integers, the set of all integers, and the set of real numbers, respectively. By a solution of (E) we mean a sequence $x: \mathbb{N} \rightarrow \mathbb{R}$ satisfying (E) for large $n$.

An important issue in the asymptotic theory of ordinary and delay differential equations is constructing sufficient conditions which ensure the existence of solutions with prescribed asymptotic behavior. From this point of view many authors studied second order differential equations with deviating argument of the form

$$
\left(r x^{\prime}\right)^{\prime}(t)=a(t) f\left(x\left(\sigma_{0}(t)\right)\right)
$$

or

$$
x^{\prime \prime}(t)=f\left(t, x\left(\sigma_{1}(t)\right), \ldots, x\left(\sigma_{m}(t)\right)\right)
$$

(C) 2018 Miskolc University Press 
where $r: \mathbb{R} \rightarrow(0,+\infty), \sigma_{i}(t) \rightarrow \infty$ as $t \rightarrow \infty$ for $i=0, \ldots, m$, see e.g. [1], [2], [3], [11], and the references therein. Since difference equations can be treated as discretization of differential equations, the existence of solutions with prescribed asymptotic behavior of difference equations was studied in literature too, see for example, in [4], [12], [13] and the references therein.

In papers [4-9], the first author presented a new theory of the study of asymptotic properties of solutions of difference equations of the form

$$
\Delta^{2} x_{n}=a_{n} f\left(x_{\sigma(n)}\right)+b_{n}
$$

in which o $\left(n^{s}\right)$, for $s \leq 0$, is used a measure of approximation. In the paper [10], we extend some of results on difference equations of Sturm-Liouville type of the form

$$
\Delta\left(r_{n} \Delta x_{n}\right)=a_{n} f\left(x_{\sigma(n)}\right)+b_{n} .
$$

This paper is a continuation of these investigations. Our main goal is to present sufficient conditions for the existence of a solution $x$ of equation (E) such that

$$
x_{n}=c\left(r_{1}^{-1}+\cdots+r_{n-1}^{-1}\right)+d+\mathrm{o}\left(n^{s}\right),
$$

where $c, d \in \mathbb{R}$ and $s \in(-\infty, 0]$. We give also sufficient conditions for a given solution $x$ of equation (E) to have an asymptotic property (1.3).

The paper is organized as follows. In Section 2, we introduce notation and present some preliminary lemmas. Next, Section 3 is devoted to our first main result Theorem 1 , some consequences of it and the example which proves that one of assumptions in main theorem is essential. In Section 4 we prove our second main result and some corollaries from it. Moreover, this section includes the example which proves that one of assumptions of main theorem is not "too" strong.

\section{PRELIMINARIES}

The space of all sequences $x: \mathbb{N} \rightarrow \mathbb{R}$ we denote by $\mathbb{R}^{\mathbb{N}}$. If $x, y$ in $\mathbb{R}^{\mathbb{N}}$, then $x y$ and $|x|$ denotes the sequences defined by $(x y)_{n}=x_{n} y_{n}$ and $|x|_{n}=\left|x_{n}\right|$, respectively. Moreover,

$$
\|x\|=\sup \left\{\left|x_{n}\right|: n \in \mathbb{N}\right\} .
$$

For any sequence $x \in \mathbb{R}^{\mathbb{N}}$ we define a sequence $x^{*}: \mathbb{N} \rightarrow \mathbb{R}^{m}$ by

$$
x_{n}^{*}= \begin{cases}(0,0, \ldots, 0) & \text { for } n<m-\sigma+1 \\ \left(x_{n+\sigma-1}, x_{n+\sigma-2}, \ldots, x_{n+\sigma-m}\right) & \text { for } n \geq m-\sigma+1 .\end{cases}
$$

We use the symbol $d_{m}$ to denote the max-metric on $\mathbb{R}^{m}$ defined by

$$
d_{m}(u, v)=\max \left\{\left|u_{1}-v_{1}\right|, \ldots,\left|u_{m}-v_{m}\right|\right\} .
$$

Moreover, $\overline{\mathrm{B}}(u, \alpha)$ denotes the closed ball of radius $\alpha$ centered at a point $u \in \mathbb{R}^{m}$. We say that a function $g: \mathbb{R}^{m} \rightarrow \mathbb{R}$ is bounded at infinity if there exists a real number $\lambda$ such that $g$ is bounded on the set

$$
[\lambda, \infty) \times \cdots \times[\lambda, \infty)=[\lambda, \infty)^{m} .
$$


In the same way, the boundedness at minus infinity can be defined.

Lemma 1. If $y \in \mathbb{R}^{\mathbb{N}}$ and $\Delta\left(r_{n} \Delta y_{n}\right)=0$, then there exist real constants $c, d$ such that

$$
y_{n}=c \sum_{j=1}^{n-1} \frac{1}{r_{j}}+d
$$

for any $n$. If $c, d \in \mathbb{R}$ and $y \in \mathbb{R}^{\mathbb{N}}$ is defined by (2.1), then $\Delta\left(r_{n} \Delta y_{n}\right)=0$.

Proof. We leave an easy proof of this lemma to the reader.

Lemma 2 ([10, Lemma 3]). Assume $s \in(-\infty, 0], t \in[s, \infty), r_{n}^{-1}=\mathrm{O}\left(n^{t}\right), a \in$ $\mathbb{R}^{\mathbb{N}}$, and

$$
\sum_{n=1}^{\infty} n^{1+t-s}\left|a_{n}\right|<\infty, \quad \text { then } \sum_{n=1}^{\infty} \frac{1}{n^{s} r_{n}} \sum_{j=n}^{\infty}\left|a_{j}\right|<\infty .
$$

Lemma 3. Assume $s \in(-\infty, 0], t \in[s, \infty), r_{n}^{-1}=\mathrm{O}\left(n^{t}\right), a \in \mathbb{R}^{\mathbb{N}}$, and at least one of the following conditions is satisfied

$$
\text { (a) } \liminf _{n \rightarrow \infty}\left(\frac{\left|a_{n}\right|}{\left|a_{n+1}\right|}-1\right)>2+t-s, \quad \text { (b) } \liminf _{n \rightarrow \infty} n \log \frac{\left|a_{n}\right|}{\left|a_{n+1}\right|}>2+t-s .
$$

Then

$$
\sum_{n=1}^{\infty} \frac{1}{n^{s} r_{n}} \sum_{j=n}^{\infty}\left|a_{j}\right|<\infty .
$$

Proof. Using [7, Lemma 6.3] in case (a), or [7, Lemma 6.4] in case (b) we obtain

$$
\sum_{n=1}^{\infty} n^{1+t-s}\left|a_{n}\right|<\infty
$$

By Lemma 2 we get the result.

Lemma 4. Assume $\lambda \in \mathbb{R}, r_{n}^{-1}=\mathrm{O}\left(n^{\lambda}\right), a \in \mathbb{R}^{\mathbb{N}}$, and at least one of the following conditions is satisfied

$$
\text { (a) } \limsup _{n \rightarrow \infty} \frac{\left|a_{n+1}\right|}{\left|a_{n}\right|}<1, \quad \text { (b) } \limsup _{n \rightarrow \infty} \sqrt[n]{\left|a_{n}\right|}<1 .
$$

Then

$$
\sum_{n=1}^{\infty} \frac{1}{n^{s} r_{n}} \sum_{j=n}^{\infty}\left|a_{j}\right|<\infty
$$

for any $s \in(-\infty, 0]$. 
Proof. Let $s \in(-\infty, 0]$. Choose a real number $t$ such that $t>\max (s, \lambda)$. Then $r_{n}^{-1}=\mathrm{O}\left(n^{t}\right)$ and, using the ratio test in case (a), or the root test in case (b) we get

$$
\sum_{n=1}^{\infty} n^{1+t-s}\left|a_{n}\right|<\infty
$$

Hence the assertion is a consequence of Lemma 2.

Lemma 5 ([10, Lemma 5]). If

$$
\sum_{k=1}^{\infty} \frac{1}{r_{k}} \sum_{i=k}^{\infty}\left|u_{i}\right|<\infty
$$

then

$$
\sum_{k=1}^{\infty}\left|u_{k}\right| \sum_{i=1}^{k} \frac{1}{r_{i}}<\infty \quad \text { and } \quad \sum_{k=n}^{\infty} \frac{1}{r_{k}} \sum_{i=k}^{\infty}\left|u_{i}\right| \leq \sum_{k=n}^{\infty}\left|u_{k}\right| \sum_{i=1}^{k} \frac{1}{r_{i}}
$$

for any $n \in \mathbb{N}$.

Lemma 6 ([4, Lemma 4.7]). Assume $y, \rho: \mathbb{N} \rightarrow \mathbb{R}$, and $\lim _{n \rightarrow \infty} \rho_{n}=0$. In the set $X=\left\{x \in \mathbb{R}^{\mathbb{N}}:|x-y| \leq|\rho|\right\}$ we define a metric by the formula

$$
d(x, z)=\|x-z\| .
$$

Then any continuous map $H: X \rightarrow X$ has a fixed point.

\section{SOLUTIONS WITH PRESCRIBED ASYMPTOTIC BEHAVIOR}

In this section we establish various conditions under which for a given solution $y$ of the equation $\Delta\left(r_{n} \Delta y_{n}\right)=0$ and a given nonpositive real $s$ there exists a solution $x$ of (E) such that $x_{n}=y_{n}+\mathrm{o}\left(n^{s}\right)$.

Theorem 1. Assume $s \in(-\infty, 0], y$ is a solution of the equation $\Delta\left(r_{n} \Delta y_{n}\right)=0$,

$$
\sum_{k=1}^{\infty} \frac{1}{k^{s} r_{k}} \sum_{j=k}^{\infty}\left(\left|a_{j}\right|+\left|b_{j}\right|\right)<\infty, \quad q \in \mathbb{N}, \quad \alpha \in(0, \infty), \quad U=\bigcup_{n=q}^{\infty} \overline{\mathrm{B}}\left(y_{n}^{*}, \alpha\right),
$$

and $f$ is continuous and bounded on $U$. Then there exists a solution $x$ of (E) such that $x_{n}=y_{n}+\mathrm{o}\left(n^{s}\right)$.

Proof. For $n \in \mathbb{N}$ and $x \in \mathbb{R}^{\mathbb{N}}$ let

$$
F(x)(n)=a_{n} f\left(x_{n}^{*}\right)+b_{n} .
$$

There exists $L>0$, such that

$$
|f(u)| \leq L
$$


for any $u \in U$. Since $s \leq 0$ we have

$$
\sum_{k=1}^{\infty} \frac{1}{r_{k}} \sum_{j=k}^{\infty}\left(L\left|a_{j}\right|+\left|b_{j}\right|\right)<\infty
$$

Let

$$
Y=\left\{x \in \mathbb{R}^{\mathbb{N}}:|x-y| \leq \alpha\right\}, \quad \rho \in \mathbb{R}^{\mathbb{N}}, \quad \rho_{n}=\sum_{k=n}^{\infty} \frac{1}{r_{k}} \sum_{j=k}^{\infty}\left(L\left|a_{j}\right|+\left|b_{j}\right|\right) .
$$

If $x \in Y$, then $x_{n}^{*} \in U$ for large $n$. Hence the sequence $\left(f\left(x_{n}^{*}\right)\right)$ is bounded for any $x \in Y$. Define sequences $w, g$ by

$$
w_{j}=L\left|a_{j}\right|+\left|b_{j}\right|, \quad g_{n}=\sum_{k=n}^{\infty} \frac{1}{k^{s} r_{k}} \sum_{j=k}^{\infty} w_{j} .
$$

Then

$$
n^{-s} \rho_{n}=n^{-s} \sum_{k=n}^{\infty} \frac{1}{r_{k}} \sum_{j=k}^{\infty} w_{j}=\sum_{k=n}^{\infty} \frac{1}{n^{s} r_{k}} \sum_{j=k}^{\infty} w_{j} \leq \sum_{k=n}^{\infty} \frac{1}{k^{s} r_{k}} \sum_{j=k}^{\infty} w_{j}=g_{n}
$$

Using the assumption

$$
\sum_{k=1}^{\infty} \frac{1}{k^{s} r_{k}} \sum_{j=k}^{\infty}\left(\left|a_{j}\right|+\left|b_{j}\right|\right)<\infty
$$

we get $g_{n}=\mathrm{o}(1)$. Hence, by (3.3),

$$
\rho_{n}=n^{s} g_{n}=n^{s} \mathrm{o}(1)=\mathrm{o}\left(n^{s}\right) .
$$

Therefore there exists an index $p$ such that

$$
\rho_{n} \leq \alpha \text { and } n+\sigma-m \geq q \text { for } n \geq p .
$$

Let

$$
\begin{gathered}
X=\left\{x \in \mathbb{R}^{\mathbb{N}}:|x-y| \leq \rho \text { and } x_{n}=y_{n} \text { for } n<p\right\}, \\
H: Y \rightarrow \mathbb{R}^{\mathbb{N}}, \quad H(x)(n)= \begin{cases}y_{n} & \text { for } n<p \\
y_{n}+\sum_{k=n}^{\infty} \frac{1}{r_{k}} \sum_{j=k}^{\infty} F(x)(j) & \text { for } n \geq p .\end{cases}
\end{gathered}
$$

Note that $X \subset Y$. If $x \in X$, then for $n \geq p$ we have

$$
\left|H(x)(n)-y_{n}\right|=\left|\sum_{k=n}^{\infty} \frac{1}{r_{k}} \sum_{j=k}^{\infty} F(x)(j)\right| \leq \sum_{k=n}^{\infty} \frac{1}{r_{k}} \sum_{j=k}^{\infty}|F(x)(j)| \leq \rho_{n} .
$$

Therefore $H X \subset X$. Let $x \in X$, and $\varepsilon>0$. By Lemma 5, we have

$$
\sum_{k=1}^{\infty}\left|a_{k}\right| \sum_{i=1}^{k} \frac{1}{r_{i}}<\infty .
$$


Choose an index $q \geq p$ and a positive constant $\gamma$ such that

$$
L \sum_{k=q}^{\infty}\left|a_{k}\right| \sum_{i=1}^{k} \frac{1}{r_{i}}<\varepsilon \quad \text { and } \quad \gamma \sum_{k=p}^{q}\left|a_{k}\right| \sum_{i=1}^{k} \frac{1}{r_{i}}<\varepsilon .
$$

Let

$$
C=\bigcup_{n=1}^{q} \overline{\mathrm{B}}\left(y_{n}^{*}, \alpha\right)
$$

Since $C$ is a compact subset of $\mathbb{R}^{m}, f$ is uniformly continuous on $C$. Choose a positive $\delta$ such that if $u_{1}, u_{2} \in C$ and $d_{m}\left(u_{1}, u_{2}\right)<\delta$, then

$$
\left|f\left(u_{1}\right)-f\left(u_{2}\right)\right|<\gamma \text {. }
$$

Choose $z \in X$ such that $\|x-z\|<\delta$. Then

$$
\begin{aligned}
& \|H x-H z\|=\sup _{n \geq p}\left|\sum_{k=n}^{\infty} \frac{1}{r_{k}} \sum_{j=k}^{\infty}(F(x)(j)-F(z)(j))\right| \\
& \leq \sum_{k=p}^{\infty} \frac{1}{r_{k}} \sum_{j=k}^{\infty}|F(x)(j)-F(z)(j)| \leq \sum_{k=p}^{\infty} \frac{1}{r_{k}} \sum_{j=k}^{\infty}\left|a_{j}\right|\left|f\left(x_{j}^{*}\right)-f\left(z_{j}^{*}\right)\right| .
\end{aligned}
$$

By Lemma 5

$$
\sum_{k=p}^{\infty} \frac{1}{r_{k}} \sum_{j=k}^{\infty}\left|a_{j}\right|\left|f\left(x_{j}^{*}\right)-f\left(z_{j}^{*}\right)\right| \leq \sum_{k=p}^{\infty}\left|a_{k}\right|\left|f\left(x_{k}^{*}\right)-f\left(z_{k}^{*}\right)\right| \sum_{i=1}^{k} \frac{1}{r_{i}} .
$$

Hence

$$
\|H x-H z\| \leq \gamma \sum_{k=p}^{q}\left|a_{k}\right| \sum_{i=1}^{k} \frac{1}{r_{i}}+2 L \sum_{k=q}^{\infty}\left|a_{k}\right| \sum_{i=1}^{k} \frac{1}{r_{i}}<3 \varepsilon .
$$

Therefore the map $H: X \rightarrow X$ is continuous with respect to the metric defined by (2.2). By Lemma 6 there exists a point $x \in X$ such that $x=H x$. Then for $n \geq p$ we have

$$
x_{n}=y_{n}+\sum_{k=n}^{\infty} \frac{1}{r_{k}} \sum_{j=k}^{\infty} F(x)(j) .
$$

Hence

$$
\Delta\left(r_{n} \Delta x_{n}\right)=\Delta\left(r_{n} \Delta y_{n}\right)+\Delta\left(r_{n} \Delta\left(\sum_{k=n}^{\infty} \frac{1}{r_{k}} \sum_{j=k}^{\infty} F(x)(j)\right)\right)
$$


for $n \geq p$. Define a sequence $G$ by

$$
G_{n}=\sum_{k=n}^{\infty} \frac{1}{r_{k}} \sum_{j=k}^{\infty} F(x)(j) .
$$

Then

$$
r_{n} \Delta G_{n}=r_{n}\left(-\frac{1}{r_{n}} \sum_{j=n}^{\infty} F(x)(j)\right),
$$

$\Delta\left(r_{n} \Delta G_{n}\right)=F(x)(n)=a_{n} f\left(x_{n}^{*}\right)+b_{n}=a_{n} f\left(x_{n+\sigma-1}, x_{n+\sigma-2}, \ldots, x_{n+\sigma-m}\right)+b_{n}$ for large $n$. Therefore $x$ is a solution of (E). Since $x \in X$ and $\rho_{n}=\mathrm{o}\left(n^{s}\right)$, we have $x_{n}=y_{n}+\mathrm{o}\left(n^{s}\right)$.

Corollary 1. Assume $f$ is continuous, $s \in(-\infty, 0]$, and

$$
\sum_{k=1}^{\infty} \frac{1}{k^{s} r_{k}} \sum_{j=k}^{\infty}\left(\left|a_{j}\right|+\left|b_{j}\right|\right)<\infty .
$$

Then for any bounded solution $y$ of the equation $\Delta\left(r_{n} \Delta y_{n}\right)=0$ there exists a solution $x$ of (E) such that $x_{n}=y_{n}+\mathrm{o}\left(n^{s}\right)$.

Proof. Assume $y$ is a bounded solution of the equation $\Delta\left(r_{n} \Delta y_{n}\right)=0$. Then the set

$$
U=\bigcup_{n=1}^{\infty} \overline{\mathrm{B}}\left(y_{n}^{*}, 1\right)
$$

is bounded. Hence $f$ is continuous and bounded on $U$. By Theorem 1 there exists a solution $x$ of (E) such that $x_{n}=y_{n}+\mathrm{o}\left(n^{s}\right)$.

Corollary 2. Assume $f$ is continuous, $s \in(-\infty, 0]$, and

$$
\sum_{k=1}^{\infty} \frac{1}{k^{s} r_{k}} \sum_{j=k}^{\infty}\left(\left|a_{j}\right|+\left|b_{j}\right|\right)<\infty .
$$

Then for any real constant $d$ there exists a solution $x$ of (E) such that $x_{n}=d+\mathrm{o}\left(n^{s}\right)$.

Proof. Any constant sequence is a bounded solution of the equation $\Delta\left(r_{n} \Delta y_{n}\right)=$ 0 . Hence the assertion is a consequence of Corollary 1.

Corollary 3. Assume $f$ is continuous, $s \in(-\infty, 0]$,

$$
\sum_{k=1}^{\infty} \frac{1}{r_{k}}<\infty, \quad \text { and } \quad \sum_{k=1}^{\infty} \frac{1}{k^{s} r_{k}} \sum_{j=k}^{\infty}\left(\left|a_{j}\right|+\left|b_{j}\right|\right)<\infty .
$$


Then for any $c, d \in \mathbb{R}$ there exists a solution $x$ of $(\mathrm{E})$ such that

$$
x_{n}=c \sum_{j=1}^{n-1} \frac{1}{r_{j}}+d+\mathrm{o}\left(n^{s}\right) .
$$

Proof. Define a sequence $y$ by

$$
y_{n}=c \sum_{j=1}^{n-1} \frac{1}{r_{j}}+d .
$$

By Lemma $1, y$ is a solution of the equation $\Delta\left(r_{n} \Delta y_{n}\right)=0$. By assumption, the sequence $y$ is bounded. Using Corollary 1 we get the result.

Corollary 4. Assume $f$ is continuous and bounded, $s \in(-\infty, 0]$, and

$$
\sum_{k=1}^{\infty} \frac{1}{k^{s} r_{k}} \sum_{j=k}^{\infty}\left(\left|a_{j}\right|+\left|b_{j}\right|\right)<\infty .
$$

Then for any solution $y$ of the equation $\Delta\left(r_{n} \Delta y_{n}\right)=0$ there exists a solution $x$ of (E) such that $x_{n}=y_{n}+\mathrm{o}\left(n^{s}\right)$.

Proof. This corollary is an immediate consequence of Theorem 1.

Corollary 5. Assume $f$ is continuous, $s \in(-\infty, 0], t \in[s, \infty), r_{n}^{-1}=\mathrm{O}\left(n^{t}\right)$, and

$$
\sum_{n=1}^{\infty} n^{1+t-s}\left(\left|a_{n}\right|+\left|b_{n}\right|\right)<\infty .
$$

Then for any bounded solution $y$ of the equation $\Delta\left(r_{n} \Delta y_{n}\right)=0$ there exists a solution $x$ of (E) such that $x_{n}=y_{n}+\mathrm{o}\left(n^{s}\right)$.

Proof. This corollary is a consequence of Lemma 2 and Corollary 1.

Corollary 6. Assume $f$ is continuous, $s \in(-\infty, 0], t \in[s, \infty), r_{n}^{-1}=\mathrm{O}\left(n^{t}\right)$, and

$$
\liminf _{n \rightarrow \infty} n\left(\frac{\left|a_{n}\right|+\left|b_{n}\right|}{\left|a_{n+1}\right|+\left|b_{n+1}\right|}-1\right)>2+t-s .
$$

Then for any bounded solution $y$ of the equation $\Delta\left(r_{n} \Delta y_{n}\right)=0$ there exists a solution $x$ of $(\mathrm{E})$ such that $x_{n}=y_{n}+\mathrm{o}\left(n^{s}\right)$.

Proof. This corollary is a consequence of Lemma 3 and Corollary 5.

Corollary 7. Assume $f$ is continuous and bounded at infinity, $\lambda \in \mathbb{R}$,

$$
\frac{1}{r_{n}}=\mathrm{O}\left(n^{\lambda}\right) \text { and } \limsup _{n \rightarrow \infty} \frac{\left|a_{n+1}\right|+\left|b_{n+1}\right|}{\left|a_{n}\right|+\left|b_{n}\right|}<1 .
$$


Then for any positive $c$, any real $d$ and any $s \in(-\infty, 0]$ there exists a solution $x$ of (E) such that

$$
x_{n}=c \sum_{j=1}^{n-1} \frac{1}{r_{j}}+d+\mathrm{o}\left(n^{s}\right) .
$$

Proof. Let $c \in(0, \infty), d \in \mathbb{R}, s \in(-\infty, 0]$. Define a sequence $y$ by

$$
y_{n}=c \sum_{j=1}^{n-1} \frac{1}{r_{j}}+d .
$$

By Lemma $1, y$ is a solution of the equation $\Delta\left(r_{n} \Delta y_{n}\right)=0$. By Lemma 4 ,

$$
\sum_{k=1}^{\infty} \frac{1}{k^{s} r_{k}} \sum_{j=k}^{\infty}\left(\left|a_{j}\right|+\left|b_{j}\right|\right)<\infty .
$$

If the series

$$
\sum_{n=1}^{\infty} \frac{1}{r_{n}}
$$

is convergent then the sequence $y$ is bounded and, using Corollary 1 we get the result. Assume the series (3.4) is divergent. Since $c>0$, we have $y_{n} \rightarrow \infty$. There exists a real number $\alpha$ such that $f$ is bounded on $[\alpha, \infty)^{m}$. There exists an index $q$ such that $y_{n+\sigma-m}>\alpha+1$ for any $n \geq q$. Let

$$
U=\bigcup_{n=q}^{\infty} \overline{\mathrm{B}}\left(y_{n}^{*}, 1\right) .
$$

Then $U \subset[\alpha, \infty)^{m}$. Hence $f$ is continuous and bounded on $U$. Now, using Theorem 1 we obtain the result.

Now we present an example that proves the assumption that the function $f$ is bounded on some "neighborhood" of $\left(y_{n}^{*}\right)$ such that $\left(y_{n}\right)$ solves $\Delta\left(r_{n} \Delta y_{n}\right)=0$ in Theorem 1, is essential.

Example 1. Assume $m=2$,

$$
r_{n}=n^{-1}, \quad a_{n}=2^{-n}, \quad b_{n}=0, \quad \sigma=0, \quad s=0, \quad f(x, y)=x^{3}+\exp (2 y) .
$$

Then equation (E) takes the form

$$
\Delta\left(n^{-1} \Delta x_{n}\right)=2^{-n}\left(x_{n-1}^{3}+\exp \left(2 x_{n-2}\right)\right) .
$$

Let

$$
y_{n}=\sum_{k=1}^{n-1} \frac{1}{r_{k}}=\sum_{k=1}^{n-1} k=\frac{n(n-1)}{2} .
$$


Then $f$ is continuous and not bounded on $\bigcup_{n=q}^{\infty} \overline{\mathrm{B}}\left(y_{n}^{*}, \alpha\right)$ for any $q \in \mathbb{N}$ and $\alpha>0$. Moreover,

$$
\sum_{k=1}^{\infty} \frac{1}{k^{s} r_{k}} \sum_{j=k}^{\infty}\left(\left|a_{j}\right|+\left|b_{j}\right|\right)=\sum_{k=1}^{\infty} k \sum_{j=k}^{\infty} \frac{1}{2^{j}}=2 \sum_{k=1}^{\infty} \frac{k}{2^{k}}<\infty .
$$

Assume $x$ is a solution of (3.5) such that

$$
x_{n}=y_{n}+z_{n},
$$

for large $n$ and $z_{n}=\mathrm{o}\left(n^{s}\right)=\mathrm{o}(1)$. Since $\Delta\left(\frac{1}{n} \Delta y_{n}\right)=0$ for large $n$, we have

$$
\Delta\left(\frac{1}{n} \Delta z_{n}\right)=\Delta\left(\frac{1}{n} \Delta x_{n}\right)=2^{-n}\left(x_{n-1}^{3}+\exp \left(2 x_{n-2}\right)\right)>0
$$

for large $n$. Therefore, the sequence $\frac{1}{n} \Delta z_{n}$ is eventually increasing, and there exists the limit

$$
\lambda=\lim _{n \rightarrow \infty} \frac{1}{n} \Delta z_{n}>-\infty .
$$

If $\lambda<\infty$, then the sequence $\frac{1}{n} \Delta z_{n}$ is convergent in $\mathbb{R}$. Hence the series

$$
\sum_{n=1}^{\infty} \Delta\left(\frac{1}{n} \Delta x_{n}\right)=\sum_{n=1}^{\infty} \Delta\left(\frac{1}{n} \Delta z_{n}\right)
$$

is convergent. On the other hand for large $n$

$$
\Delta\left(\frac{1}{n} \Delta x_{n}\right)=2^{-n}\left(x_{n-1}^{3}+\exp \left(2 x_{n-2}\right)\right) \geq 2^{-n} \exp \left(2 x_{n-2}\right) .
$$

Since

$$
x_{n} \geq \frac{y_{n}}{2}
$$

for large $n$, we get that

$$
\Delta\left(\frac{1}{n} \Delta z_{n}\right)>2^{-n} \exp ((n-2)(n-3)) .
$$

Hence $\lambda=\infty$. Therefore $\frac{1}{n} \Delta z_{n}>1$ for large $n$ and we get

$$
\sum_{n=1}^{\infty} \Delta z_{n} \geq \sum_{n=1}^{\infty} n=\infty
$$

On the other hand, since $z_{n} \rightarrow 0$, the series $\sum_{n=1}^{\infty} \Delta z_{n}$ is convergent. 


\section{ASYMPTOTIC BEHAVIOR OF SOLUTIONS}

In this section we present sufficient conditions for a given solution $x$ of equation (E) to have an asymptotic property $x_{n}=y_{n}+\mathrm{o}\left(n^{s}\right)$, where $y$ is a solution of the equation $\Delta\left(r_{n} \Delta y_{n}\right)=0$ and $s \in(-\infty, 0]$.

Theorem 2. Assume $x$ is a solution of (E) such that the sequence $\left(f\left(x_{n}^{*}\right)\right)$ is bounded,

$$
s \in(-\infty, 0], \text { and } \sum_{k=1}^{\infty} \frac{1}{k^{s} r_{k}} \sum_{j=k}^{\infty}\left(\left|a_{j}\right|+\left|b_{j}\right|\right)<\infty .
$$

Then there exists a solution $y$ of the equation $\Delta\left(r_{n} \Delta y_{n}\right)=0$ such that

$$
x_{n}=y_{n}+\mathrm{o}\left(n^{s}\right) \text {. }
$$

Proof. Define a sequence $u$ by

$$
u_{n}=\Delta\left(r_{n} \Delta x_{n}\right)=a_{n} f\left(x_{n}^{*}\right)+b_{n} .
$$

Since the sequence $\left(f\left(x_{n}^{*}\right)\right)$ is bounded, we have

$$
\sum_{k=1}^{\infty} \frac{1}{k^{s} r_{k}} \sum_{j=k}^{\infty}\left|u_{j}\right|<\infty
$$

Define sequences $w, y, z$ by

$$
w_{n}=\sum_{k=n}^{\infty} \frac{1}{r_{k}} \sum_{j=k}^{\infty} u_{j}, \quad y_{n}=x_{n}-w_{n}, \quad z_{n}=\sum_{k=n}^{\infty} \frac{1}{k^{s} r_{k}} \sum_{j=k}^{\infty} u_{j} .
$$

Then

$$
n^{-s}\left|w_{n}\right| \leq n^{-s} \sum_{k=n}^{\infty} \frac{1}{r_{k}} \sum_{j=k}^{\infty}\left|u_{j}\right|=\sum_{k=n}^{\infty} \frac{1}{n^{s} r_{k}} \sum_{j=k}^{\infty}\left|u_{j}\right| \leq \sum_{k=n}^{\infty} \frac{1}{k^{s} r_{k}} \sum_{j=k}^{\infty}\left|u_{j}\right|=\mathrm{o}(1) .
$$

Hence $w_{n}=\mathrm{o}\left(n^{S}\right)$. Moreover

$$
\Delta\left(r_{n} \Delta w_{n}\right)=\Delta\left(r_{n} \Delta\left(\sum_{k=n}^{\infty} \frac{1}{r_{k}} \sum_{j=k}^{\infty} u_{j}\right)\right)=-\Delta\left(r_{n}\left(\frac{1}{r_{n}} \sum_{j=n}^{\infty} u_{j}\right)\right)=u_{n}
$$

and we obtain

$\Delta\left(r_{n} \Delta y_{n}\right)=\Delta\left(r_{n} \Delta x_{n}\right)-\Delta\left(r_{n} \Delta w_{n}\right)=u_{n}-u_{n}=0, \quad x_{n}=y_{n}+w_{n}=y_{n}+\mathrm{o}\left(n^{s}\right)$.

Corollary 8. Assume $f$ is locally bounded, $s \in(-\infty, 0]$, and

$$
\sum_{k=1}^{\infty} \frac{1}{k^{s} r_{k}} \sum_{j=k}^{\infty}\left(\left|a_{j}\right|+\left|b_{j}\right|\right)<\infty \text {. }
$$


Then for any bounded solution $x$ of (E) there exists a solution $y$ of the equation

$$
\Delta\left(r_{n} \Delta y_{n}\right)=0
$$

such that $x_{n}=y_{n}+\mathrm{o}\left(n^{s}\right)$.

Proof. Assume $x$ is a bounded solution of (E). Then the sequence $x^{*}$ is also bounded. Since $f$ is locally bounded, the sequence $\left(f\left(x_{n}^{*}\right)\right)$ is bounded. Hence the result follows from Theorem 2 .

Corollary 9. Assume $f$ is bounded, $s \in(-\infty, 0]$, and

$$
\sum_{k=1}^{\infty} \frac{1}{k^{s} r_{k}} \sum_{j=k}^{\infty}\left(\left|a_{j}\right|+\left|b_{j}\right|\right)<\infty .
$$

Then for any solution $x$ of $(\mathrm{E})$ there exists a solution $y$ of the equation

$$
\Delta\left(r_{n} \Delta y_{n}\right)=0
$$

such that $x_{n}=y_{n}+\mathrm{o}\left(n^{s}\right)$.

Proof. The assertion is an immediate consequence of Theorem 2.

Corollary 10. Assume $f$ is bounded and

$$
\sum_{k=1}^{\infty}\left(\frac{1}{r_{k}}+\left|a_{k}\right|+\left|b_{k}\right|\right)<\infty .
$$

Then any solution $x$ of $(\mathrm{E})$ is convergent.

Proof. Let $s=0$ and let $x$ be a solution of (E). By assumption the series $\sum_{k=1}^{\infty} 1 / r_{k}$ is convergent and the sequence $u$ defined by $u_{k}=\sum_{j=k}^{\infty}\left(\left|a_{j}\right|+\left|b_{j}\right|\right)$ is convergent to zero. Hence

$$
\sum_{k=1}^{\infty} \frac{1}{r_{k}} \sum_{j=k}^{\infty}\left(\left|a_{j}\right|+\left|b_{j}\right|\right)<\infty .
$$

Therefore, by Corollary 9 and Lemma 1, there exist real constants $c, d$ such that

$$
x_{n}=c \sum_{j=1}^{n-1} \frac{1}{r_{j}}+d+\mathrm{o}\left(n^{s}\right) .
$$

Since $s=0$ and $\sum_{k=1}^{\infty} 1 / r_{k}<\infty$, we get $\lim _{n \rightarrow \infty} x_{n}=c \sum_{k=1}^{\infty} 1 / r_{k}+d$.

Corollary 11. Assume $f$ is locally bounded and

$$
\sum_{k=1}^{\infty}\left(\frac{1}{r_{k}}+\left|a_{k}\right|+\left|b_{k}\right|\right)<\infty .
$$

Then any bounded solution $x$ of $(\mathrm{E})$ is convergent. 
Proof. Let $s=0$ and let $x$ be a bounded solution of (E). As in the proof of Corollary 10 we obtain (4.1). Using Corollary 8 and Lemma 1 we get the result.

Corollary 12. Assume $f$ is locally bounded, $s \in(-\infty, 0], t \in[s, \infty), r_{n}^{-1}=\mathrm{O}\left(n^{t}\right)$, and

$$
\sum_{n=1}^{\infty} n^{1+t-s}\left(\left|a_{n}\right|+\left|b_{n}\right|\right)<\infty .
$$

Then for any bounded solution $x$ of (E) there exists a solution $y$ of the equation $\Delta\left(r_{n} \Delta y_{n}\right)=0$ such that $x_{n}=y_{n}+\mathrm{o}\left(n^{s}\right)$.

Proof. The assertion is a consequence of Lemma 2 and Corollary 8.

Corollary 13. Assume $f$ is locally bounded, $s \in(-\infty, 0], t \in[s, \infty), r_{n}^{-1}=\mathrm{O}\left(n^{t}\right)$,

$$
\sum_{n=1}^{\infty} \frac{1}{r_{n}}=\infty, \quad \text { and } \sum_{n=1}^{\infty} n^{1+t-s}\left(\left|a_{n}\right|+\left|b_{n}\right|\right)<\infty .
$$

Then for any bounded solution $x$ of (E) there exists a real constant $d$ such that

$$
x_{n}=d+\mathrm{o}\left(n^{s}\right) \text {. }
$$

Proof. Assume $x$ be a bounded solution of (E). By Lemma 2 and Corollary 8, there exist $c, d \in \mathbb{R}$ such that

$$
x_{n}=c \sum_{j=1}^{n-1} \frac{1}{r_{j}}+d+\mathrm{o}\left(n^{s}\right) .
$$

Since $x$ is bounded and $\sum_{n=1}^{\infty} 1 / r_{n}=\infty$, we have $c=0$.

Corollary 14. Assume $f$ is locally bounded,

$$
\frac{1}{r_{n}}=\mathrm{O}(1), \text { and } \sum_{n=1}^{\infty} n\left(\left|a_{n}\right|+\left|b_{n}\right|\right)<\infty .
$$

Then any bounded solution of (E) is convergent.

Proof. Let $t=s=0$ and let $x$ be a bounded solution of (E). By Corollary 12 there exists a solution $y$ of the equation $\Delta\left(r_{n} \Delta y_{n}\right)=0$ such that $x_{n}=y_{n}+\mathrm{o}(1)$. Then $y$ is a bounded sequence. By Lemma 1 any bounded solution $y$ of the equation $\Delta\left(r_{n} \Delta y_{n}\right)=0$ is convergent. Hence $x$ is convergent.

Corollary 15. Assume $f$ is bounded at infinity, $s \in(-\infty, 0], t \in[s, \infty), r_{n}^{-1}=$ $\mathrm{O}\left(n^{t}\right)$,

$$
\liminf _{n \rightarrow \infty} n \log \frac{\left|a_{n}\right|+\left|b_{n}\right|}{\left|a_{n+1}\right|+\left|b_{n+1}\right|}>2+t-s,
$$

and $x$ is a solution of (E) such that $\lim _{n \rightarrow \infty} x_{n}=\infty$. Then there exists a solution $y$ of the equation $\Delta\left(r_{n} \Delta y_{n}\right)=0$ such that $x_{n}=y_{n}+\mathrm{o}\left(n^{s}\right)$. 
Proof. Since $f$ is bounded at infinity and $\lim _{n \rightarrow \infty} x_{n}=\infty$, the sequence $\left(f\left(x_{n}^{*}\right)\right)$ is bounded. Using Lemma 3 and Theorem 2 we get the result.

Corollary 16. Assume $f$ is locally bounded, $\lambda \in \mathbb{R}, r_{n}^{-1}=\mathrm{O}\left(n^{\lambda}\right)$,

$$
\limsup _{n \rightarrow \infty} \frac{\left|a_{n+1}\right|+\left|b_{n+1}\right|}{\left|a_{n}\right|+\left|b_{n}\right|}<1,
$$

and $x$ is a bounded solution of (E). Then for any $s \in(-\infty, 0]$ there exists a solution $y$ of the equation $\Delta\left(r_{n} \Delta y_{n}\right)=0$ such that $x_{n}=y_{n}+\mathrm{o}\left(n^{s}\right)$.

Proof. This assertion is a consequence of Lemma 4 and Corollary 8.

Now we present an example that proves the assumption

$$
\sum_{k=1}^{\infty} \frac{1}{k^{s} r_{k}} \sum_{j=k}^{\infty}\left|a_{j}\right|<\infty
$$

is not enough in Theorem 2.

Example 2. Assume $m=2, s=0, \sigma=0$,

$$
r_{n}=n^{2}, \quad a_{n}=\frac{1}{n^{2}}, \quad b_{n}=2 n+1-\frac{2}{n^{2}}, \quad f(x, y)=\frac{x}{|x|+1}+\frac{y+3}{|y|+2} .
$$

Then equation $(\mathrm{E})$ takes the form

$$
\Delta\left(n^{2} \Delta x_{n}\right)=\frac{1}{n^{2}}\left(\frac{x_{n-1}}{\left|x_{n-1}\right|+1}+\frac{x_{n-2}+3}{\left|x_{n-2}\right|+2}\right)+2 n+1-\frac{2}{n^{2}} .
$$

Notice that $f$ is bounded and

$$
\sum_{k=1}^{\infty} \frac{1}{k^{s} r_{k}} \sum_{j=k}^{\infty}\left|a_{j}\right|=\sum_{k=1}^{\infty} \frac{1}{k^{2}} \sum_{j=k}^{\infty} \frac{1}{j^{2}}<\infty
$$

Moreover, the sequence $x_{n}=n$, is a solution of (4.2). On the other hand, any solution of the equation $\Delta\left(n^{2} \Delta y_{n}\right)=0$ is of the form

$$
y_{n}=c \sum_{k=1}^{n-1} \frac{1}{r_{k}}+d=c \sum_{k=1}^{n-1} \frac{1}{k^{2}}+d
$$

for some $c, d \in \mathbb{R}$. Hence any solution of $\Delta\left(n^{2} \Delta y_{n}\right)=0$ is convergent, which means that $x$ cannot be approximated by any solution of the equation $\Delta\left(n^{2} \Delta y_{n}\right)=0$. 


\section{REFERENCES}

[1] R. P. Agarwal, S. Djebali, T. Moussaoui, and O. G. Mustafa, "On the asymptotic integration of nonlinear differential equations," J. Comput. Appl. Mathematics, vol. 202, pp. 352-376, 2007, doi: 10.1016/j.cam.2005.11.038.

[2] M. Bohner and S. Stević, "Asymptotic behavior of second-order dynamic equations," Appl. Math. Comput., vol. 188, no. 2, pp. 1503-1512, 2007, doi: 10.1016/j.amc.2006.11.016.

[3] O. Lipovan, "On the asymptotic behavior of the solutions to a class of second order nonlinear differential equations," Glasgow Math. J., vol. 45, no. 2, pp. 179-187, 2003, doi: 10.1017/S0017089502001143.

[4] J. Migda, "Approximative solutions of difference equations," Electron. J. Qual. Theory Differ. Equ., no. 13, pp. 1-26, 2014, doi: 10.14232/ejqtde.2014.1.13.

[5] J. Migda, "Iterated remainder operator, tests for multiple convergence of series and solutions of difference equations," Adv. Difference Equ., no. 189, pp. 1-18, 2014, doi: 10.1186/1687-18472014-189.

[6] J. Migda, "Approximative solutions to difference equations of neutral type," Appl. Math. Comput., vol. 268, pp. 763-774, 2015, doi: 10.1016/j.amc.2015.06.097.

[7] J. Migda, "Qualitative approximation of solutions to difference equations," Electron. J. Qual. Theory Differ. Equ., no. 32, pp. 1-26, 2015, doi: 10.14232/ejqtde.2015.1.32.

[8] J. Migda, "Mezocontinuous operators and solutions of difference equations," Electron. J. Qual. Theory Differ. Equ., no. 11, pp. 1-16, 2016, doi: 10.14232/ejqtde.2016.1.11.

[9] J. Migda and M. Migda, "Qualitative approximation of solutions to discrete Volterra equations," Electron. J. Qual. Theory Differ. Equ., no. 3, pp. 1-27, 2018, doi: 10.14232/ejqtde.2018.1.3.

[10] J. Migda and M. Nockowska-Rosiak, "Asymptotic properties of solutions to difference equations of Sturm-Liouville type," Appl. Math. Comput., vol. 340, pp. 126-137, 2019, doi: 10.1016/j.amc.2018.08.001.

[11] C. G. Philos, "Positive increasing solutions on the half-line to second order nonlinear delay differential equations," Glasgow Math. J., vol. 49, pp. 197-211, 2007, doi: 10.1017/S0017089507003667.

[12] P. Rehák, "Asymptotic formulae for solutions of linear second order difference equations," $J$. Difference Equ. Appl., vol. 22, no. 1, pp. 107-139, 2016, doi: 10.1080/10236198.2015.1077815.

[13] S. Stević, "Asymptotic behaviour of second-order difference equation," ANZIAM J., vol. 46, no. 1, pp. 157-170, 2004, doi: 10.1017/S1446181100013742.

Authors' addresses

Janusz Migda

Faculty of Mathematics and Computer Science, A. Mickiewicz University, Umultowska 87, 61-614 Poznań, Poland

E-mail address: migda@amu.edu.pl

Magdalena Nockowska-Rosiak

Institute of Mathematics, Lodz University of Technology, Wólczańska 215, 90-924 Łódź, Poland

E-mail address: magdalena.nockowskaep.lodz.pl 